\title{
Sacralization may be associated with facet orientation and tropism but not degenerative changes of the lumbar vertebrae
}

\author{
Tuba Selcuk Can ${ }^{A, B, D, E, F}$, Behice Kaniye Yilmaz ${ }^{B, D, F}$, Sevim Ozdemir ${ }^{B, D, E}$ \\ Department of Radiology, Istanbul Haseki Training and Research Hospital, Istanbul, Turkey
}

\section{Abstract}

\begin{abstract}
Purpose: In this retrospective study, we aimed to investigate the possible effects of transitional vertebra anatomy on facet joint tropism and orientation by evaluating lumbar magnetic resonance imaging (MRI) studies performed at our institution.
\end{abstract}

\begin{abstract}
Material and methods: We included 84 patients with sacralization of the L5 vertebra and an equal number of patients with a radiology report within normal limits as the control group in our study. We compared facet tropism (FT) and orientation between both groups.

Results: In both the sacralization group and the control group, the facet orientation angle showed a significant increasing trend from the L1-L2 level to the L5-S1 level $(p<0.001)$. The orientation angle of the L5-S1 level was higher in the sacralization group compared to the control group $(p<0.01)$. In the evaluation of FJ orientation between the sacralization and control groups, we found that coronal orientation was significantly more frequent at the L5-S1 level in the sacralization group. When the 2 groups were compared with regard to tropism at each spinal level, the sacralization group had a significantly higher FT frequency at the L5-S1 level $(p<0.001)$.

Conclusions: To our knowledge, this is the first study to evaluate the relationship between sacralization and facet joint tropism. However, there were no relationships between facet degeneration, disc degeneration/herniation, and sacralization. Our results indicate that, although patients with sacralization and controls had similar characteristics in most assessments, they demonstrated significant differences at the L5-S1 level in terms of orientation and tropism.
\end{abstract}

Key words: sacralization, facet orientation, tropism.

\section{Introduction}

The anterior intervertebral discs and posterior facet joints (FJs) form the functional structure of the spine [1]. FJs are critical because they carry approximately $16 \%$ of the vertical load on the lumbar vertebrae and they play a role in degenerative diseases of the lumbar spine [2-4]. Facet tropism (FT) is the asymmetry between the right and left FJ angles, and it contributes to degeneration via alteration of lumbar spine biomechanics $[5,6]$. The FJ orientation is transverse according to the coronal plane, and its relationship with degenerative spondylolisthesis has been demon- strated in several studies [7,8]. In a finite-element study by Kim et al., it was suggested that the sagittal orientation of FJs predisposes the spine to spondylolisthesis, and FT presence increases stress due to asymmetric forces in the related segment [9].

Lumbosacral transitional vertebrae (LSTV) are a common congenital anomaly of the lumbosacral junction. It can be observed as either unilateral or bilateral assimilation of the L5 vertebra with the sacrum or ilium (sacralization) or the appearance of the first segment of the sacral vertebra (S1) as a part of the lumbar vertebrae (lumbarization) [10]. Several studies have investigated the relationship between

\section{Correspondence address:}

Tuba Selcuk Can, MD, S.B.U Haseki Egitim Ve Arastirma Hastanesi, Radyoloji Klinigi, Haseki Sultan Mah., Adnan Adivar Cad. 34096 Fatih/Istanbul, Turkey

phone:+905327843477, e-mail:drtubas@gmail.com

Authors' contribution:

A Study design · B Data collection · C Statistical analysis · D Data interpretation · E Manuscript preparation · F Literature search · G Funds collection 
LSTV and low back pain and degenerative disc disease; while some studies reported a relationship [2,11], others did not reveal any [5]. Differences in LSTV anatomy can alter the distribution of load on FJs in the lumbosacral junction may predispose the spine to degeneration [12]. In this study, we aimed to compare individuals with sacralization of the L5 vertebra and those with normal lumbosacral junction anatomy in terms of possible differences in FT and orientation, and to determine the possible effects of sacralization and its asymmetric nature on FJ tropism. In addition, we evaluated whether the facet orientation angle was associated with the levels of facet degeneration and disc degeneration or herniation.

\section{Material and methods}

\section{Study population}

Ethics committee approval was obtained for this study. Informed consent was obtained from each participant included in the study. A total of 874 consecutive lumbar magnetic resonance (MR) imaging examinations performed at our clinic from July 2018 to November 2018 for the evaluation of lumbar radicular pain and low back pain were retrospectively reviewed. Patients with lumbar surgical history, trauma, spinal inflammation, infectious disease, spinal malignancy, or scoliosis were excluded from the study to avoid spinal anatomy changes associated with these conditions. Whole spine MR images were used to number the lumbar vertebrae by craniocaudally counting from $\mathrm{C} 2$ and cross-referencing lumbar vertebra sagittal MR images. A total of 23 patients with lumbarization of $S 1$ (defined as the separation of S1 from the sacral vertebrae) [10] were excluded. A total of 84 pa-

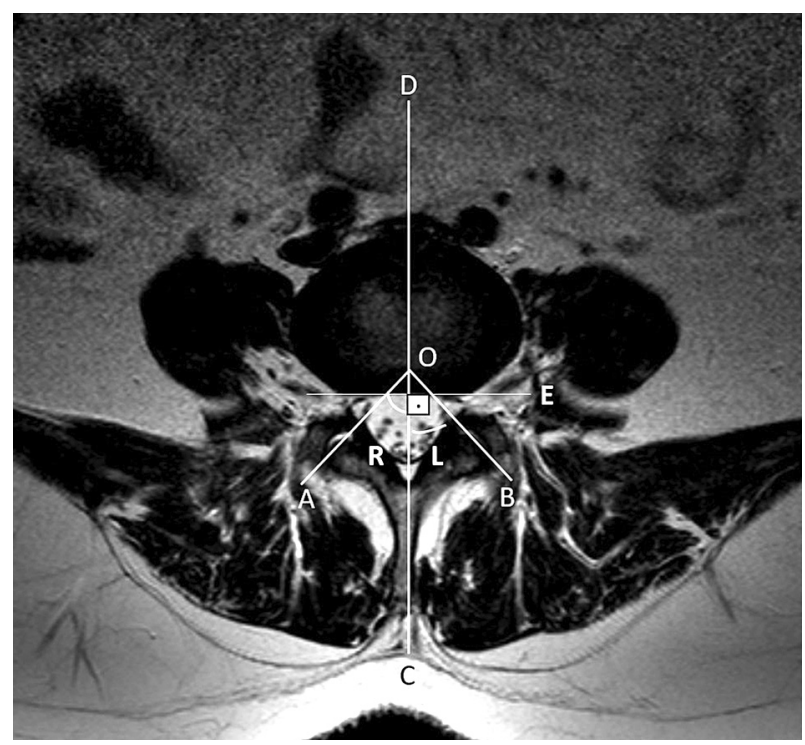

Figure 1. Facet orientation angle measurement. DC is the reference line passing through the spinous process and perpendicular to the line $E$ tangent to the disc posterior margin. The mean value of the angles between $A 0-D C$ and $\mathrm{BO}-\mathrm{DC}(\mathrm{R}+\mathrm{L})$ is the orientation angle for this level tients with sacralization of the L5 vertebra transverse process, defined as the unilateral or bilateral fusion with the sacrum, were identified by the evaluation of axial and survey MR images. A control group comprising 84 patients with normal lumbosacral junction anatomy, matched for age and gender distribution, were included in the study.

\section{Imaging protocol and landmark definitions}

All lumbar MR images were obtained according to a standard lumbar MR imaging protocol (axial T2-weighted fast spin-echo, TR: $3800 \mathrm{~ms}$ and TE: $100 \mathrm{~ms}$; sagittal T2-weighted fast spin-echo, TR: $2500 \mathrm{~ms}$ and TE: $100 \mathrm{~ms}$; and sagittal T1-weighted spin-echo, TR: $500 \mathrm{~ms}$ and TE: $10 \mathrm{~ms}$ ) with the use of a 1.5 Tesla Philips Achieva MRI device (Philips Healthcare, Best, Netherlands). Other matrix parameters were as follows: 256 X 192, section thickness: $3 \mathrm{~mm}$, interslice gap: $4 \mathrm{~mm}$, FOV: $22 \mathrm{~cm}$ (axial image) and $28 \mathrm{~cm}$ (sagittal images). Images were evaluated on a workstation (INFINITT PACS, Infinitt Healthcare, South Korea).

For the facet orientation angle, axial T2-weighted images parallel to the endplate at the mid-disc level were used. The line passing through the centre of the disc space and the spinous process basis was accepted as a reference. The mean value of the angle between the reference line and the lines passing through the anteromedial and posterolateral margins of the superior articular facets on both sides was considered as the facet orientation angle for that level [6,11] (Figure 1). Accordingly, when the angle was $>45^{\circ}$, the orientation was considered to be coronal, and when the angle was $\leq 45^{\circ}$, the orientation was considered to be sagittal [1].

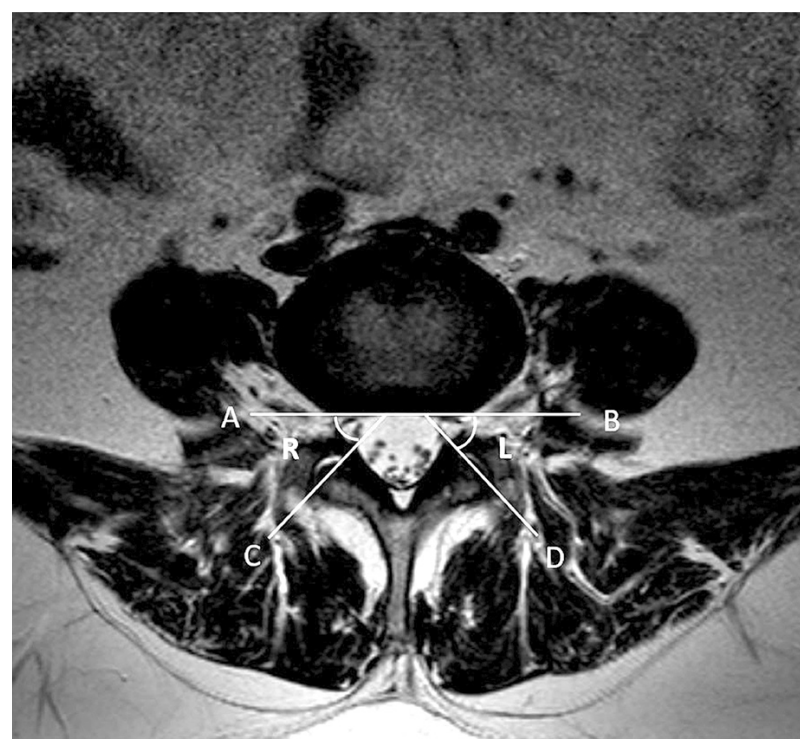

Figure 2. Facet tropism measurement. $A B$ is the reference line. The angle between $A B$ and line $6 C$ is the right facet angle $(R)$, and the angle between $A B$ and line $D$ is the left facet angle $(L)$ 


\section{Evaluation of images}

To evaluate FT, axial T2-weighted MR images passing through the most caudal slice of the intervertebral disc space (in which the FJs were most likely to be bisected) were used. The line tangent to the posterior disc was considered as the reference line. The angle between the reference line and the line passing through the anteromedial and posterolateral margins of the superior articular facets was measured bilaterally. If the difference between the angle for the right facet and the angle for the left facet was greater than $10^{\circ}$, tropism was considered to be present (Figure 2). Because FJs have greater coronal orientation at lower vertebral levels when the right-left difference is positive, we assumed that the right FJ had a more sagittal orientation and called it right FT, and when this value was negative, we assumed that the left FJ had a more sagittal orientation and called it left FT.

We also evaluated the presence of degenerative disc disease in the lower lumbar spinal segments of patients with sacralization (and its possible relationships with tropism). Sacralization type (left-sided, right-sided, or both) were also taken into account. For the evaluation of disc degeneration, we identified regions demonstrating altered signal intensity and/or disc herniation of any form (i.e. protrusion, prolapse, extrusion, or sequestration). Degeneration was defined as when the signal intensity of the intervertebral disc was lower than the cerebrospinal fluid or when it showed nonuniform signal intensity. The degree of degeneration and herniation were graded from 1 to 4 [13]. All FJs were also evaluated with respect to degeneration findings and were scored from 1 to 4 (1: normal, 2: minor narrowing in joint distance, 3: sclerosis and small osteophytes, 4: pronounced osteophytes).

\section{Statistical analysis}

Statistical analyses of the data were performed with the Statistical Package for the Social Sciences version 15.0. The data for continuous variables were expressed as mean \pm standard deviation, and the data for categorical variables were expressed as frequency and percentage. To compare the distributions of categorical variables between the 2 groups, $\chi^{2}$ tests and Fisher's exact tests were used. For continuous variables, Student's $t$-test and the MannWhitney $U$ test were used regarding parametric/nonparametric assumptions. To compare the 5 vertebral levels, the Friedman test was used. The results were evaluated with a 95\% confidence interval and a significance level of $p<0.05\left({ }^{*} p<0.05,{ }^{* *} p<0.01\right.$, and $\left.{ }^{* * *} p<0.001\right)$. Interobserver agreement was evaluated with Cohen's $\kappa$ coefficient (values $\leq 0$ : no agreement, 0.01-0.20: none to slight, 0.21-0.40: fair, 0.41-0.60: moderate, 0.61-0.80: substantial, and 0.81-1.00: almost perfect agreement).

\section{Results}

The prevalence of sacralization was found to be $9.6 \%$ in our study. The ages of individuals in the sacralization group ranged between 19 and 59 years, with a mean age of $36.89 \pm 11.76$ years. The ages of individuals in the control group ranged between 20 and 55 years, with a mean age of $34.27 \pm 7.91$ years. There was no statistically significant difference between the 2 groups in terms of age $(p=0.092)$.

In both the sacralization group and the control group, the facet orientation angle showed a significant increasing trend from the L1-L2 level to the L5-S1 level ( $p<0.001)$. Except for the L5-S1 level, there were no significant differences between the orientation angles of the control and sacralization groups. The orientation angle of the L5-S1 level was higher in the sacralization group compared to the control group $(p<0.01)$. In the evaluation of FJ orientation between the sacralization and control groups, we found that coronal orientation was significantly more frequent at the L5-S1 level in the sacralization group. The orientations of the FJs were similar for other levels (Table 1).

The frequency of tropism presence at any level (overall) was $79.8 \%(n=67)$ in the sacralization group and $73.8 \%(n=62)$ in the control group; statistical analysis showed that there was no statistically significant difference between groups $(p=0.361)$. When the 2 groups were compared regarding tropism at each spinal level, the sacralization group had a significantly higher FT frequency at the L5-S1 level $(p<0.001)$ (Table 2).

When the side of sacralization and FT at the L5-S1 level was evaluated, right-sided tropism was found in $55.6 \%$ of cases with right-sided sacralization. Left-sided tropism was found in $45.5 \%$ of cases with left-sided sacralization. Additionally, $51.5 \%$ of patients with bilateral sacralization had no tropism. Among patients with sacralization, the presence of degenerative disc disease in the relevant segments according to the presence/absence of tropism (for L3-L4, L4-L5 or L5-S1) is shown in Table 3. The differences were not statistically significant among those with and without tropism $(p>0.05)$.

When we analysed whether facet joint orientation values were associated with the degree of facet joint degeneration in each respective level, we found that there were no relationships between controls or patients and sacralization (Table 4). Further analysis of whether the degree of facet degeneration was associated with sacralization type (left-sided, right-sided or both) also yielded non-significant results.

Next, we evaluated the possible relationship between degree of disc herniation/degeneration and facet joint orientation angle. The results did not show any significant findings with respect to any of the levels (Table 5).

Interobserver agreement values for the measurement of the FJ orientation angle and tropism angle were 0.85 (range: 0.82-0.89) and 0.89 (range: 0.86-0.94), respectively. 
Table 1. Facet joint orientations to lumbar levels in the sacralization and control group

\begin{tabular}{|c|c|c|c|c|c|}
\hline \multirow[t]{2}{*}{ Facet orientation } & \multicolumn{2}{|c|}{ Sacralization group } & \multicolumn{2}{|c|}{ Control group } & \multirow[t]{2}{*}{$p(1)$} \\
\hline & $n$ & $\%$ & $n$ & $\%$ & \\
\hline \multicolumn{6}{|l|}{ L1-I2 } \\
\hline Sagittal & 84 & 100.0 & 82 & 97.6 & \multirow[t]{2}{*}{0.497} \\
\hline Coronal & 0 & 0.0 & 2 & 2.4 & \\
\hline \multicolumn{6}{|l|}{ L2-I3 } \\
\hline Sagittal & 83 & 98.8 & 80 & 95.2 & \multirow[t]{2}{*}{0.367} \\
\hline Coronal & 1 & 1.2 & 4 & 4.8 & \\
\hline \multicolumn{6}{|l|}{ L3-I4 } \\
\hline Sagittal & 70 & 83.3 & 69 & 82.1 & \multirow[t]{2}{*}{0.838} \\
\hline Coronal & 14 & 16.7 & 15 & 17.9 & \\
\hline \multicolumn{6}{|l|}{ L4-I5 } \\
\hline Sagittal & 47 & 56.0 & 43 & 51.2 & \multirow[t]{2}{*}{0.536} \\
\hline Coronal & 37 & 44.0 & 41 & 48.8 & \\
\hline \multicolumn{6}{|l|}{ L5-s1 } \\
\hline Sagittal & 21 & 25.0 & 35 & 41.7 & \multirow[t]{2}{*}{$0.022^{*}$} \\
\hline Coronal & 63 & 75.0 & 49 & 58.3 & \\
\hline
\end{tabular}

${ }^{*} p<0.05 . p(1): p$-value for comparison of sacralization and control groups

Table 2. Facet tropism according to groups and levels

\begin{tabular}{|c|c|c|c|c|c|}
\hline \multirow[t]{2}{*}{ Tropism } & \multicolumn{2}{|c|}{ Sacralization group } & \multicolumn{2}{|c|}{ Control group } & \multirow[t]{2}{*}{$p(1)$} \\
\hline & $n$ & $\%$ & $n$ & $\%$ & \\
\hline \multicolumn{6}{|l|}{ L1-L2 } \\
\hline$(-)$ & 65 & 77.4 & 66 & 78.6 & \multirow[t]{2}{*}{0.852} \\
\hline$(+)$ & 19 & 22.6 & 18 & 21.4 & \\
\hline \multicolumn{6}{|l|}{ L2-L3 } \\
\hline$(-)$ & 69 & 82.1 & 64 & 76.2 & \multirow[t]{2}{*}{0.342} \\
\hline$(+)$ & 15 & 17.9 & 20 & 23.8 & \\
\hline \multicolumn{6}{|l|}{ L3-L4 } \\
\hline$(-)$ & 68 & 81.0 & 60 & 71.4 & \multirow[t]{2}{*}{0.147} \\
\hline$(+)$ & 16 & 19.0 & 24 & 28.6 & \\
\hline \multicolumn{6}{|l|}{ L4-L5 } \\
\hline$(-)$ & 60 & 71.4 & 64 & 76.2 & \multirow[t]{2}{*}{0.483} \\
\hline$(+)$ & 24 & 28.6 & 20 & 23.8 & \\
\hline \multicolumn{6}{|l|}{ L5-S1 } \\
\hline$(-)$ & 38 & 45.2 & 63 & 75.0 & \multirow[t]{2}{*}{$0.0001^{* * *}$} \\
\hline$(+)$ & 46 & 54.8 & 21 & 25.0 & \\
\hline
\end{tabular}

${ }^{* * *} p<0.001 . p(1)$ : $p$-value for comparison of sacralization and control groups

\section{Discussion}

The FJs act as essential posterior stabilization mechanisms during movement of the spine [6], and the importance of FJ orientation in this function has been demonstrated in various studies $[6,14]$. Researchers have been interested in the relationship between facet orientation and degenerative spine disease; however, findings have often been contradictory $[1,7,15]$. Although the presence of LSTV affects the dynamics of the lumbosacral junction, until today, there have been very few studies that evaluated FJ orientation at each lumbar level in patients with LSTV. This study showed 
Table 3. Disc herniation by disc degeneration according to tropism status and levels in sacralization group

\begin{tabular}{|c|c|c|c|c|c|}
\hline \multirow[t]{2}{*}{ Tropism } & \multicolumn{2}{|c|}{ Tropism (-) } & \multicolumn{2}{|c|}{ Tropism (+) } & \multirow[t]{2}{*}{$p$-value } \\
\hline & $n$ & $\%$ & $n$ & $\%$ & \\
\hline \multicolumn{6}{|l|}{ L3-L4 } \\
\hline \multicolumn{6}{|c|}{ Disc degeneration } \\
\hline$(-)$ & 48 & 70.6 & 13 & 81.3 & \multirow[t]{2}{*}{0.538} \\
\hline$(+)$ & 20 & 29.4 & 3 & 18.8 & \\
\hline \multicolumn{6}{|c|}{ Disc herniation } \\
\hline$(-)$ & 61 & 89.7 & 15 & 93.8 & \multirow[t]{2}{*}{0.999} \\
\hline$(+)$ & 7 & 10.3 & 1 & 6.3 & \\
\hline \multicolumn{6}{|l|}{ L4-L5 } \\
\hline \multicolumn{6}{|c|}{ Disc degeneration } \\
\hline$(-)$ & 22 & 36.7 & 11 & 45.8 & \multirow[t]{2}{*}{0.437} \\
\hline+$)$ & 38 & 63.3 & 13 & 54.2 & \\
\hline \multicolumn{6}{|c|}{ Disc herniation } \\
\hline$(-)$ & 34 & 56.7 & 16 & 66.7 & \multirow[t]{2}{*}{0.399} \\
\hline$(+)$ & 26 & 43.3 & 8 & 33.3 & \\
\hline \multicolumn{6}{|l|}{ L5-S1 } \\
\hline \multicolumn{6}{|c|}{ Disc degeneration } \\
\hline$(-)$ & 32 & 84.2 & 41 & 89.1 & \multirow[t]{2}{*}{0.534} \\
\hline$(+)$ & 6 & 15.8 & 5 & 10.9 & \\
\hline \multicolumn{6}{|c|}{ Disc herniation } \\
\hline$(-)$ & 38 & 100.0 & 45 & 97.8 & \multirow[t]{2}{*}{0.999} \\
\hline$(+)$ & 0 & 0.0 & 1 & 2.2 & \\
\hline
\end{tabular}

Table 4. Relationships between facet joint orientation angle and facet degeneration at each level in both groups

\begin{tabular}{|l|c|c|c|c|}
\hline \multirow{2}{*}{ L1-L2 Orientation angle } & \multicolumn{2}{|c|}{ Patients with sacralization } & \multicolumn{2}{c|}{ Patients without sacralization } \\
& $\begin{array}{l}\text { Left facet degeneration } \\
\end{array}$ & Right facet degeneration & Left facet degeneration & Right facet degeneration \\
& $p=0.026$ & $r=-0.027$ & $r=0.035$ & $r=-0.049$ \\
\hline L2-L3 Orientation angle & $r=0.082$ & $p=0.769$ & $p=0.701$ & $p=0.587$ \\
& $p=0.366$ & $r=-0.126$ & $r=0.112$ & $r=-0.148$ \\
& $r=-0.139$ & $p=0.165$ & $p=0.217$ & $p=0.104$ \\
\hline L3-L4 Orientation angle & $p=0.115$ & $r=-0.119$ & $r=0.020$ & $r=-0.019$ \\
& $r=-0.048$ & $p=0.188$ & $p=0.820$ & $p=0.834$ \\
\hline L4-L5 Orientation angle & $p=0.590$ & $r=-0.033$ & $r=0.014$ & $p=0.015$ \\
& $r=0.104$ & $p=0.702$ & $p=0.871$ & $r=0.869$ \\
\hline L5-S1 Orientation angle & $p=0.223$ & $r=0.051$ & $r=0.015$ & $p=0.739$ \\
\hline
\end{tabular}

Kendal's tau-b statistic

that the orientation angle demonstrates a marked increase from L1 to S1 in patients with LSTV, as well as healthy subjects; the latter finding is supported by a study by Wang et al. [6]. Despite this similarity between the 2 groups, our findings showed that the orientation angle was significantly higher at the L5-S1 level in patients with LSTV when compared to controls, indicating a differentiating characteristic.
The broader and horizontal orientation at the L5-S1 level compared to other segments in patients with sacralization may be caused by the primary anatomical difference in these subjects - the fusion of the transverse process of the L5 vertebra with the sacrum.

In terms of disc degeneration, our study did not reveal any significant relationship between imaging findings of 
Table 5. Relationships between disc generation/herniation and facet joint orientation angle at each level in both groups.Kendal's tau-b statistic

\begin{tabular}{|l|c|c|c|c|}
\hline & \multicolumn{2}{|c|}{ Patients with sacralization } & \multicolumn{2}{c|}{ Patients without sacralization } \\
& Disc degeneration & Disc herniation & Disc degeneration & Disc herniation \\
\hline \multirow{2}{*}{ L1-L2 Orientation angle } & $r=-0.103$ & $r=-0.022$ & $r=-0.152$ & $r=0.069$ \\
& $p=0.257$ & $p=0.809$ & $p=0.094$ & $p=0.445$ \\
\hline L2-L3 Orientation angle & $r=-0.019$ & $r=-0.043$ & $r=-0.111$ & $r=-0.016$ \\
& $p=0.842$ & $p=0.638$ & $p=0.223$ & $p=0.856$ \\
\hline L3-L4 Orientation angle & $r=-0.039$ & $r=0.054$ & $r=0.146$ & $r=0.015$ \\
& $p=0.660$ & $p=0.548$ & $p=0.107$ & $p=0.866$ \\
\hline L4-L5 Orientation angle & $r=0.023$ & $r=-0.010$ & $r=0.046$ & $r=-0.042$ \\
& $p=0.790$ & $p=0.911$ & $p=0.603$ & $p=0.639$ \\
\hline L5-S1 Orientation angle & $r=0.041$ & $r=0.017$ & $r=-0.051$ & $r=0.003$ \\
& $p=0.648$ & $p=0.853$ & $p=0.568$ & $p=0.977$ \\
\hline
\end{tabular}

degeneration and the presence/absence of tropism. Interestingly, Jentzsch et al. [1] reported that sagittal orientation of the lower lumbar levels was associated with degeneration of the FJs. Fujiwara et al. [16] also obtained similar results with an MRI study. When our findings and the results of these 2 studies are taken together, it appears that coronal orientation of the lower lumbar levels may be protective against degenerative changes.

In a study by Kim et al. [9] that discussed the biomechanical effect of FT it was found that the presence of FT in the related segment increased stress on the disc and also facet contact force. The effects of this asymmetry in the FJs with regard to degenerative spine disease has been demonstrated in different studies $[17,18]$. Similarly, the effects of LSTV on low back pain and degenerative spine disease have been a subject of curiosity for several authors. In a study by Sekharappa et al., degenerative disc disease was more common at the L5-S1 level compared to other levels [14]. Various studies have implicated LSTV as a cause of low back pain [19], degenerative changes [20,21], and other spinal abnormalities [20]. However, despite demonstrating an increased tendency for low back pain in patients with LSTV, Gopalan et al. [19] suggested that the higher likelihood of other spinal abnormalities in patients with LSTV was in fact the cause of low back pain, rather than the presence of LSTV alone. This was in support of several studies, one of which had found a higher frequency of degenerative changes without any association with low back pain in individuals with LSTV [21]. However, an earlier study by Vergauwen et al., which utilized a prospective design, reported no difference among controls and patients with LSTV in terms of the frequency of degenerative spinal changes. Despite this overall similarity between groups, the spinal level above the LSTV was determined to have a higher frequency of degenerative findings when compared to healthy subjects [22]. Considering the conclusions of newer studies in addition to the earlier report by Vergauwen et al., we believe that current evidence shows a lack of a direct relationship between LSTV and degenerative changes or lower back pain. Although further studies are necessary to draw definite conclusions on this matter, our results suggest that the spinal changes in patients with LSTV may be a physiological response aimed at preventing injury. However, these changes no doubt result in altered biomechanics, and thus may predispose individuals to problems of the lumbar spine in the future.

In this study, in which the presence of tropism at all lumbar levels in LSTV patients was evaluated, our results showed that the tropism frequency was $54.8 \%$ at the L5$\mathrm{S} 1$ level, and comparisons revealed that tropism was significantly more common in patients with sacralization compared to the control group (25\%). In addition, lower lumbar level FJs had a higher frequency of coronal orientation in our study, and degenerative disc diseases at these levels did not demonstrate a significant difference from the control group. FT was more prominent in patients with sacralization than controls; however, these features did not translate to a higher frequency of degeneration. As mentioned previously, this phenomenon may be a mechanism of adaptation to sacralization of the FJs, thereby preventing degenerative changes in the lumbar spine. However, future studies would benefit from longitudinal analyses to ascertain whether such adaptations lead to spinal problems with advanced age.

To ascertain whether there were any relationships between facet joint orientation and clinically relevant findings, such as facet degeneration, disc degeneration and disc hernia, we performed correlation analyses, which demonstrated that facet joint orientation was not associated with any of the analysed parameters.

The most significant limitation of our study is that the control group was comprised of symptomatic patients, even though they had normal lumbosacral junction anatomy. Another limitation is that patients' BMIs were not included in the evaluation. We only included patients with sacralization rather than including all types of LSTV to predict the influence of the unilateral or bilateral nature of abnormal articulation on FT. Additionally, because we included patients based on sacralization and not the presence/absence of pathological states (degeneration, herniation etc.), the analysis of correlations between these characteristics and 
facet joint orientation may not reflect the alterations in patients with significant pathology; thus, these results cannot be generalized to those with disc-related pathologies. We believe there is a need for future studies to assess these relationships in patients with and without clinical pathologies, possibly with the inclusion of clinical characteristics as secondary parameters. Finally, because the study was retrospective, evaluation of patients' daily physical activities was not possible.

Despite these limitations, to our knowledge, this study is the first to evaluate the relationship between sacralization and FT at all lumbar levels. Our results indicate that, although patients with sacralization and controls had similar characteristics in most assessments, they demon- strated significant differences at the L5-S1 level in terms of orientation and tropism. Although these changes would fundamentally alter the biomechanics of this critical area, the fact that degenerative changes were not associated with tropism or facet orientation indicates that these alterations may be considered as physiological tolerance mechanisms rather than pathophysiological alterations, even though it is crucial to ascertain the longitudinal results of these changes with long-term studies.

\section{Conflict of interest}

The authors report no conflict of interest.

\section{References}

1. Jentzsch T, Geiger J, Zimmermann SM, et al. Lumbar facet joint arthritis is associated with more coronal orientation of the facet joints at the upper lumbar spine. Radiol Res Pract 2013; 2013: 693971.

2. Kalichman L, Hunter DJ. Lumbar facet joint osteoarthritis: a review. Semin Arthritis Rheum 2007; 37: 69-80.

3. Boden SD, Riew KD, Yamaguchi K, et al. Orientation of the lumbar facet joints: association with degenerative disc disease. J Bone Joint Surg Am 1996; 78: 403-411.

4. Gao T, Lai Q, Zhou S, et al. Correlation between facet tropism and lumbar degenerative disease: a retrospective analysis. BMC Musculoskelet Disord 2017; 18: 483.

5. Tulsi RS, Hermanis GM. A study of the angle of inclination and facet curvature of superior lumbar zygapophyseal facets. Spine (Phila Pa 1976) 1993; 18: 1311-1317.

6. Wang $\mathrm{H}$, Zhang Z, Zhou Y. Irregular alteration of facet orientation in lumbar segments: possible role in pathology of lumbar disc herniation in adolescents. World Neurosurg 2016; 86: 321-327.

7. Kalichman L, Suri P, Guermazi A, et al. Facet orientation and tropism: associations with facet joint osteoarthritis and degenerative spondylolisthesis. Spine (Phila Pa 1976) 2009; 34: E579.

8. Toyone T, Ozawa T, Kamikawa K, et al. Facet joint orientation difference between cephalad and caudad portions: a possible cause of degenerative spondylolisthesis. Spine (Phila Pa 1976) 2009; 34: 2259-2262.

9. Kim H-J, Chun H-J, Lee H-M, et al. The biomechanical influence of the facet joint orientation and the facet tropism in the lumbar spine. Spine J 2013; 13: 1301-1308.

10. Konin G, Walz D. Lumbosacral transitional vertebrae: classification, imaging findings, and clinical relevance. Am J Neuroradiol 2010; 31: 1778-1786.

11. Chadha M, Sharma G, Arora SS, et al. Association of facet tropism with lumbar disc herniation. Eur Spine J 2013; 22: 1045-1052.

12. Mahato NK. Facet dimensions, orientation, and symmetry at L5-S1 junction in lumbosacral transitional states. Spine (Phila Pa 1976) 2011; 36: E569-E573.
13. Schleich C, Müller-Lutz A, Blum K, et al. Facet tropism and facet joint orientation: risk factors for the development of early biochemical alterations of lumbar intervertebral discs. Osteoarthritis Cartilage 2016; 24: 1761-1768.

14. Sekharappa V, Amritanand R, Venkatesh Krishnan KSD. Lumbosacral transition vertebra: prevalence and its significance. Asian Spine J 2014; 8: 51-58.

15. Kong MH, He W, Tsai YD, et al. Relationship of facet tropism with degeneration and stability of functional spinal unit. Yonsei Med J 2009; 50: 624-629.

16. Fujiwara A, Tamai K, An HS, et al. Orientation and osteoarthritis of the lumbar facet joint. Clin Orthop Relat Res 2001; 385: 88-94.

17. Dai L, Jia L. Role of facet asymmetry in lumbar spine disorders. Acta Orthop Belg 1996; 62: 90-93.

18. Karacan I, Aydin T, Sahin Z, et al. Facet angles in lumbar disc herniation: their relation to anthropometric features. Spine (Phila Pa 1976) 2004; 29: 1132-1136.

19. Gopalan B, Yerramshetty JS. Lumbosacral transitional vertebra-related low back pain: resolving the controversy. Asian Spine J 2018; 12: 407-415.

20. Abbas J, Peled N, Hershkovitz I, et al. Is lumbosacral transitional vertebra associated with degenerative lumbar spinal stenosis? Biomed Res Int 2019; 2019: 3871819.

21. Luoma K, Vehmas T, Raininko R, et al. Lumbosacral transitional vertebra: relation to disc degeneration and low back pain. Spine (Phila $\mathrm{Pa}$ 1976) 2004; 29: 200-205.

22. Vergauwen S, Parizel PM, Van Breusegem L, et al. Distribution and incidence of degenerative spine changes in patients with a lumbo-sacral transitional vertebra. Eur Spine J 1997; 6: 168-172. 